\title{
Peer Support for Self-Management of Chronic Pain: the Evaluation of a Peer Coach-Led Intervention to Improve Pain Symptoms (ECLIPSE) Trial
}

\author{
Marianne S. Matthias, PhD ${ }^{1,2,3}$, Matthew J. Bair, MD, MS $S^{1,2,3}$, Susan Ofner, $M S^{4}$, \\ Michele Heisler, MD ${ }^{5,6}$, Marina Kukla, $\mathrm{PhD}^{1,7}$, Alan B. McGuire, PhD ${ }^{1,7}$, \\ Jasma Adams, $\mathrm{MPH}^{7}$, Carol Kempf, $R N^{1,3}$, Emilee Pierce, $B S^{1,2}$, Tetla Menen ${ }^{7}$, \\ Stephanie McCalley ${ }^{7}$, Nicole L. Johnson, $\mathrm{MA}^{7}$, and Joanne Daggy, PhD
}

\begin{abstract}
${ }^{7} V A$ HSR\&D Center for Health Information and Communication, Roudebush VA Medical Center Indianapolis, IN, USA; ${ }^{2}$ Regenstrief Institutelndianapolis, IN, USA; ${ }^{3}$ Department of Medicine, Indiana University of Medicinelndianapolis, IN, USA; ${ }^{4}$ Department of Biostatistics, Indiana University School of Medicinelndianapolis, IN, USA; ${ }^{5}$ Department of Internal Medicine, University of MichiganAnn Arbor, MI, USA; ${ }^{6}$ VA HSR\&D Center for Clinical Management ResearchAnn Arbor, MI, USA; ${ }^{7}$ Department of Psychology, Indiana University-Purdue University Indianapolislndianapolis, IN, USA.
\end{abstract}

BACKGROUND: Pain self-management is an effective, evidence-based treatment for chronic pain. Peer support, in which patients serve as coaches for other patients, has been effective in other chronic conditions and is a potentially promising approach to implementing pain selfmanagement programs using fewer clinical resources.

OBJECTIVE: To test a peer coach-delivered pain selfmanagement program for chronic pain.

DESIGN: Randomized controlled trial.

PARTICIPANTS: Veterans with chronic musculoskeletal pain.

INTERVENTION: Intervention patients were assigned a trained peer coach for 6 months. Coaches, who were volunteers, were asked to contact their assigned patients, either by phone or in person, twice per month. Coaches and patients were given an intervention manual to guide sessions. The control group was offered a 2 -hour pain selfmanagement class.

MAIN MEASURES: The primary outcome was total pain, assessed by the Brief Pain Inventory (BPI). Secondary outcomes were anxiety, depression, pain catastrophizing, self-efficacy, social support, patient activation, healthrelated quality of life, and healthcare utilization. Outcomes were measured at baseline, 6 months, and 9 months.

KEY RESULTS: Two hundred fifteen patients enrolled (120 intervention, 95 control). Adherence to intervention protocol was low, with only $13 \%$ of patients reporting having at least the recommended 12 peer coach meetings over the 6-month intervention. BPI total decreased from baseline to 6 months and baseline to 9 months in both groups. At 9 months, this change was statistically significant (intervention, $-0.40, p=0.018$; control, $-0.47, p=$ 0.006). There was not a statistically significant difference between groups on BPI at either time point. No secondary

Electronic supplementary material The online version of this article (https://doi.org/10.1007/s11606-020-06007-6) contains supplementary material. which is available to authorized users.

Received February 10, 2020

Accepted June 23, 2020

Published online July 22, 2020 outcomes improved significantly in either group after adjusting for multiple comparisons.

CONCLUSIONS: Patients randomized to peer support did not differ from control patients on primary and secondary outcomes. Other peer support models that do not rely on volunteers might be more effective.

TRIAL REGISTRATION: ClinicalTrials.gov Identifier: NCT02380690

KEY WORDS: ECLIPSE; chronic pain; peer support; pain selfmanagement.

J Gen Intern Med 35(12):3525-33

DOI: $10.1007 /$ s11606-020-06007-6

(C) Society of General Internal Medicine (This is a U.S. government work and not under copyright protection in the U.S.; foreign copyright protection may apply) 2020

\section{BACKGROUND}

Chronic pain affects approximately 100 million Americans and is estimated to cost $\$ 560-635$ billion annually in direct medical costs and lost worker productivity. ${ }^{1}$ Chronic pain, like other chronic conditions, requires effective and consistent selfmanagement for optimal outcomes. Self-management has been defined as "the ability to manage the symptoms, treatment, physical and psychosocial consequences and life-style changes inherent in living with a chronic condition" ${ }^{22}$ and has been recognized by the National Academy of Medicine and the Veterans Health Administration (VHA) as a critical ingredient in chronic pain management. ${ }^{1}$ For patients with chronic pain, self-management involves a combination of treatment adherence, behavioral change, adapting life roles, managing negative emotions, and coping skills.

In addition to pain self-management activities such as stretching, exercise, and modification of activities, patients frequently need motivation and encouragement in their daily efforts to self-manage. In prior studies patients have indicated difficulty in adhering to their daily self-management activities, 
identifying lack of support and lack of motivation to maintain self-management activities as key barriers to adherence. In contrast, patients have indicated that in addition to the selfmanagement activities themselves, they value having someone to help them troubleshoot when self-management activities do not seem to help, to listen to them when they want to talk about their pain or frustrations with self-management, and to simply provide reinforcement and encouragement to "keep going" with their self-management activities. ${ }^{3-5}$

Much of the self-management support identified in the above studies came from healthcare professionals who were part of studies involving a pain self-management intervention. In those studies, nurse care managers had regular contacts with patients, delivering self-management information; assisting with goal setting; and providing encouragement, accountability, and motivation. However, nurses and other care providers are not always readily available given other clinic responsibilities. Using peer coaches to provide support to patients managing chronic pain represents a promising means to deliver many of the same benefits as a nurse, but with greater implementation potential, since patients with chronic pain are more plentiful than nurses. Using peer coaches in such a role is an innovative approach to increasing the reach and adoption of pain self-management, potentially benefitting a greater number of patients with chronic pain.

Peer support models are increasingly being used to help patients manage chronic conditions and have shown promising results. Peer support involves "lay individuals with experiential knowledge who extend natural (embedded) social networks and complement professional health services." ${ }^{, 6}$ Peer support in diabetes care has resulted in significantly lower hemoglobin A1c levels, increased diabetes-specific social support, and increased self-efficacy. ${ }^{7-9}$ In a mental health setting, compared with those in usual care, patients served by a case management team that included a peer support specialist improved significantly more on patient activation (self-management self-efficacy). ${ }^{10}$ This is important, since highly activated patients with chronic conditions are more likely than less activated patients to adhere to treatment recommendations and self-management activities and are more likely to report better experiences with care and care coordination. ${ }^{1-13}$

Despite the positive effects of peer support in chronic disease self-management, research has largely neglected peer support among patients with chronic pain. This is an important research gap, given the high potential for effectiveness and implementation, since patients with pain are numerous and "graduates" of a peer support program can in turn mentor other patients, producing a self-perpetuating program with longterm sustainability. Evaluation of a peer Coach-Led Intervention to improve Pain Symptoms (ECLIPSE) is a randomized controlled trial designed to test the effects of peer-supported pain self-management on pain and secondary outcomes of self-efficacy, social support, pain catastrophizing, patient activation, health-related quality of life, and health service utilization. We hypothesized that patients randomized to the peer support arm would experience reduced pain (severity and interference) and improved secondary outcomes compared with patients randomized to the control group.

\section{METHODS}

ECLIPSE compared a 6-month peer coaching self-management intervention to a control group consisting of a 2-hour pain selfmanagement class. This control group was chosen because our purpose was to understand the role of peer support in facilitating self-management, rather than to understand the effects of selfmanagement itself. Thus, the control class covered selfmanagement topics but did not offer ongoing contact, support, or encouragement to patients. Participants were recruited from August 2015 to August 2018 and were veterans receiving care from one of six primary care clinics at a Midwestern VA Medical Center. Details of the trial protocol have previously been described. ${ }^{14}$

\section{Intervention}

A detailed description of the ECLIPSE intervention is available elsewhere. ${ }^{14}$ In brief, 68 peer coaches were enrolled. After a 2to 3-h training session, peer coaches were assigned patients matched on gender and, when possible, pain location. Each coach was assigned one patient to start, and coaches could choose how many patients they wanted to mentor. Almost half (47\%) chose to coach just one patient. With the patient's permission, coaches were given their contact information and asked to call their assigned patient. Coach-patient pairs chose whether they wanted to meet in person or via telephone and were asked to meet/talk at least two times per month, for a total of 12 sessions over the 6-month intervention period. Check-in calls were conducted by study staff to ensure peer coaches and patients made contact and to assist when contact had not been made. Coaches and patients were guided by a manual focused on pain self-management, although coaches were instructed to be flexible and were encouraged to discuss their personal experiences with pain self-management and how they overcame challenges. Manual topics are covered in detail elsewhere ${ }^{14}$ but include relaxation skills, activity pacing, cognitive behavioral skills, and self-care skills. In addition, coaches were asked to participate in monthly "booster" sessions to reinforce coaching skills, answer questions, and troubleshoot problems. Coaches were not compensated for sessions but were compensated for completing outcome assessments (see the "Outcome Measures" section). Patients randomized to the control group were offered a 2-hour pain self-management class, which covered the same general topics listed above.

\section{Participants}

Eligible patients met the following criteria: (1) musculoskeletal pain in the low back, cervical spine, or extremities (hip, knee, or shoulder) for $\geq 3$ months; (2) at least moderate pain 
severity, defined by pain $\geq 5$ on a 0 (no pain) to 10 (worst pain imaginable) scale; and (3) willingness to engage in phone or in-person contact on a regular basis with another patient. Patients were excluded if the electronic medical record review indicated (1) psychiatric hospitalization in the last 6 months; (2) current substance dependence; (3) severe medical conditions precluding participation (e.g., New York Heart Association Class III or IV heart failure); (4) if the eligibility screener given to prospective participants revealed active suicidal ideation, severe hearing or speech impairment, or pending surgery for a musculoskeletal condition (e.g., back surgery); or (5) current participation in another pain study. Primary care providers granted permission to recruit their patients. Peer coaches had musculoskeletal pain in the low back, cervical spine, or extremities (hip, knee, or shoulder) for $\geq 3$ months and were either completers of a prior pain self-management intervention, recommended by their primary care providers because their providers believed that they were successful pain self-managers, or completers of the ECLIPSE intervention as a patient.

\section{Randomization}

Following the baseline interview, participants were randomized to either the intervention or control group. To obtain random treatment assignment for the 215 patients ( 95 control, 120 intervention), permuted block randomization was used so that within each block the allocation ratio was maintained (19 control group: 24 intervention group).

\section{Outcome Measures}

Assessments were conducted at baseline, 6 months, and 9 months and administered to both patient participants and peer coaches, who were compensated $\$ 30$ per completed assessment. The primary outcome measure was the Brief Pain Inventory (BPI) total score, which assesses pain severity and impact on function and is scored on a $0-10$ scale with higher scores representing more severe and functionally limiting pain. Demographic measures and baseline primary and secondary outcome measures are summarized in Table 1 and described in detail elsewhere. ${ }^{14}$ Healthcare utilization measures were also considered secondary outcomes and were obtained through chart review. These measures included phone/secure messaging, outpatient visits, emergency department visits, hospitalizations, hospital days, and opioid medications during the 9-month study.

\section{Statistical Analysis}

Sample size was determined to ensure adequate power for the primary hypothesis that patients randomized to peer support will experience greater improvement in pain (BPI total) than patients in the control arm at the 6-month endpoint. ECLIPSE was powered to detect a small to medium effect size of $0.45 .{ }^{15}$

Primary analyses employed an intent-to-treat approach. Baseline patient characteristics were compared using appropriate test statistics (Chi-square test, Fisher's exact test, $t$-tests, or Wilcoxon rank-sum tests) to verify that randomization

Table 1 Baseline characteristics

\begin{tabular}{|c|c|c|c|c|c|}
\hline & Inter & $=119)$ & Con & & $p$ Value \\
\hline Demographic, no. (\%) & & & & & \\
\hline Age, mean (SD), years & 55.4 & (12.6) & 58.6 & $(13.3)$ & 0.074 \\
\hline Male & 95 & $(79.8)$ & 78 & $(83.0)$ & 0.559 \\
\hline White race & 76 & $(63.9)$ & 55 & $(58.5)$ & 0.339 \\
\hline Hispanic & 2 & $(1.7)$ & 4 & $(4.3)$ & $0.409 *$ \\
\hline Married/partner & 58 & $(49.2)$ & 52 & $(55.3)$ & 0.221 \\
\hline Education $>$ high school & 90 & (76.3) & 73 & $(77.7)$ & 0.812 \\
\hline Income comfortable** & 61 & $(51.3)$ & 40 & $(42.6)$ & $0.172 *$ \\
\hline Employed or retired & 85 & $(72.0)$ & 70 & $(74.5)$ & 0.691 \\
\hline Pain scores (range), mean (SD) & & & & & \\
\hline BPI total (range, $0-10$ [worst]) & 5.8 & (1.9) & 5.8 & (1.9) & 0.799 \\
\hline BPI severity (range, $0-10$ [worst]) & 6.2 & (1.5) & 5.9 & (1.7) & 0.312 \\
\hline BPI interference (range, $0-10$ [worst]) & 5.7 & $(2.5)$ & 5.7 & (2.4) & 0.956 \\
\hline Secondary outcomes & & & & & \\
\hline Perceived social support (range, 12-24 [best]) & 62.2 & $(16.5)$ & 62.6 & $(18.1)$ & 0.862 \\
\hline Self-efficacy (range, $0-10$ [best]) & 6.1 & $(2.2)$ & 6.2 & $(2.3)$ & 0.556 \\
\hline Patient activation measure (range, $0-100$ [best]) & 60.6 & $(13.5)$ & 57.9 & $(13.8)$ & 0.160 \\
\hline GAD-7 anxiety (range, $0-21$ [worst]) & 6.1 & (5.3) & 6.2 & $(5.4)$ & 0.843 \\
\hline PHQ-8 depression (range, $0-24$ [worst]) & 9.3 & (6.4) & 8.9 & $(6.0)$ & 0.634 \\
\hline Pain Catastrophizing Scale (range, $0-52$ [worst]) & 20.8 & $(13.1)$ & 21.5 & (13.3) & 0.694 \\
\hline SF-36 (range, $0-100$ [best]) & & & & & \\
\hline General health perceptions & 52.2 & (20.9) & 52.8 & $(21.1)$ & 0.836 \\
\hline Physical functioning & 45.1 & $(25.6)$ & 42.0 & $(22.0)$ & 0.350 \\
\hline Role limits due to physical health & 27.1 & $(35.9)$ & 19.9 & $(32.7)$ & 0.132 \\
\hline Role limits due to emotional health & 56.4 & $(43.0)$ & 55.6 & $(44.3)$ & 0.888 \\
\hline Energy fatigue & 39.5 & $(24.7)$ & 38.0 & (21.4) & 0.638 \\
\hline Emotional well-being & 67.9 & $(23.1)$ & 70.4 & (20.2) & 0.415 \\
\hline Social functioning & 57.4 & $(30.6)$ & 57.7 & $(29.0)$ & 0.953 \\
\hline Bodily pain & 37.6 & (21.9) & 39.4 & (19.4) & 0.540 \\
\hline
\end{tabular}

*Fisher's exact test

**Patients were asked if their income allowed them to be comfortable, was just enough to make ends meet, or was not enough to make ends meet 
achieved balanced groups. For the primary outcome of total BPI score, a linear mixed model was fit and included fixed effects of time as categorical, group, and the time $\mathrm{X}$ group interaction. Random effects included a random patientspecific intercept and a random effect for peer-coach in the intervention group only to account for the potential correlation of outcomes from patients assigned the same peer coach. ${ }^{16}$ The primary contrast of interest is the difference in change from baseline at 6 months between the two treatment arms. For BPI subscales of pain severity and interference as well as secondary outcomes, a Sidak adjustment was used to account for multiple comparisons at a given time point.

For healthcare utilization measures (phone/secure messaging, outpatient visits, emergency department visits, hospitalizations, hospital days, and opioid use over the 9-month study period), a generalized linear mixed model was used, assuming that the counts follow a negative binomial distribution with a group indicator as the only fixed effect. All analyses were conducted in SAS V9.4 (Cary, NC) with significance level set at 0.05 .

\section{Exploratory Analyses}

Intervention Dose. The number of meetings with the peer coach was self-reported by the patient and was available for 84 intervention participants $(70.5 \%)$. Fifty-four patients $(64 \%)$ had 5 or fewer contacts with their peer coach. To ascertain whether an intervention effect was present among patients who had closer to the recommended 12 contacts, we focused on the 30 patients who had at least 6 peer coach meetings. Toward this end, the primary linear mixed models were fit to this subset of patients and all control patients.

Moderation. Baseline patient activation was explored as a potential moderator of the intervention effect using the linear mixed models for the primary and secondary outcomes with the addition of all 2- and 3-way interactions between the potential moderator (patient activation), group, and time. Moderation occurs if the 3-way interaction is statistically significant.

\section{RESULTS}

The current paper focuses on the effects of peer coaching on patients with chronic pain. Peer coach outcomes are reported elsewhere. ${ }^{17}$ Figure 1 depicts the participant flow in ECLIPSE. Of the 274 patients determined to be eligible, 215 enrolled in the trial, with 120 to be randomized to the peer coaching intervention and 95 to the control group. However, two patients who enrolled withdrew prior to completing baseline assessments, leaving 119 randomized to the intervention group and 94 to the control group.
Randomization resulted in both groups being balanced on baseline characteristics (Table 1). The total sample had a mean age of 56.7 years; $80.8 \%$ were male; $62 \%$ were White, $27.7 \%$ African-American, and the remainder were other races. Baseline pain was of moderate severity.

Although peer coach-patient pairs were instructed to meet twice monthly, for a total of 12 contacts, only 11 (13.1\%) patients (of the 84 for whom data are available) met with their coaches 12 or more times during the 6-month intervention. Thirty patients $(35.7 \%)$ met at least 6 times during the intervention; the remainder $(63 \%)$ met 5 or fewer times, with 10 patients $(8 \%)$ reporting no meetings with their peer coaches. Only 6 dyads met exclusively in person. The remainder used phone or a combination of phone and in-person meetings. For patients randomized to the control group, 34 (36\%) attended the pain self-management class.

\section{Primary Outcome}

There was not a statistically significant difference between intervention and control groups in the primary outcome at 6 months. BPI total decreased from baseline to 6 months and baseline to 9 months in both groups. At 9 months, this decrease was statistically significant for both groups (intervention, $-0.40, p=0.018$; control, $-0.47, p=0.006$ ) (see Table 2).

In terms of reaching a clinically meaningful reduction in pain (defined as a $30 \%$ or greater reduction in $\mathrm{BPI}^{18}$ ) at 6 months, $19.57 \%$ of intervention patients and $27.91 \%$ of control patients experienced clinically meaningful decreases. At 9 months, $34.5 \%$ and $37.8 \%$, respectively, experienced clinically meaningful reductions.

\section{BPI Severity and Interference Subscales}

BPI severity decreased significantly in the intervention group from baseline to 6 months $(-0.35$, adjusted $p=0.048)$ and baseline to 9 months $(-0.42$, adjusted $p=0.019)$. The control group did not experience a significant decrease from baseline to 6 months $(-0.18$, adjusted $p=0.449)$, and the decrease from baseline to 9 months fell just short of significance (0.36 , adjusted $p=0.057$ ). The between-group difference was not statistically significant.

The only statistically significant decrease in BPI interference was the baseline to 9-month change in the control group $(-0.57$, adjusted $p=0.015)$ (see Table 2).

\section{Secondary Outcomes}

No secondary outcomes improved significantly in either group after adjusting for multiple comparisons (see Table 2).

\section{Healthcare Utilization}

There were no significant differences in the number of phone and secure messages, ED visits, outpatient visits, 


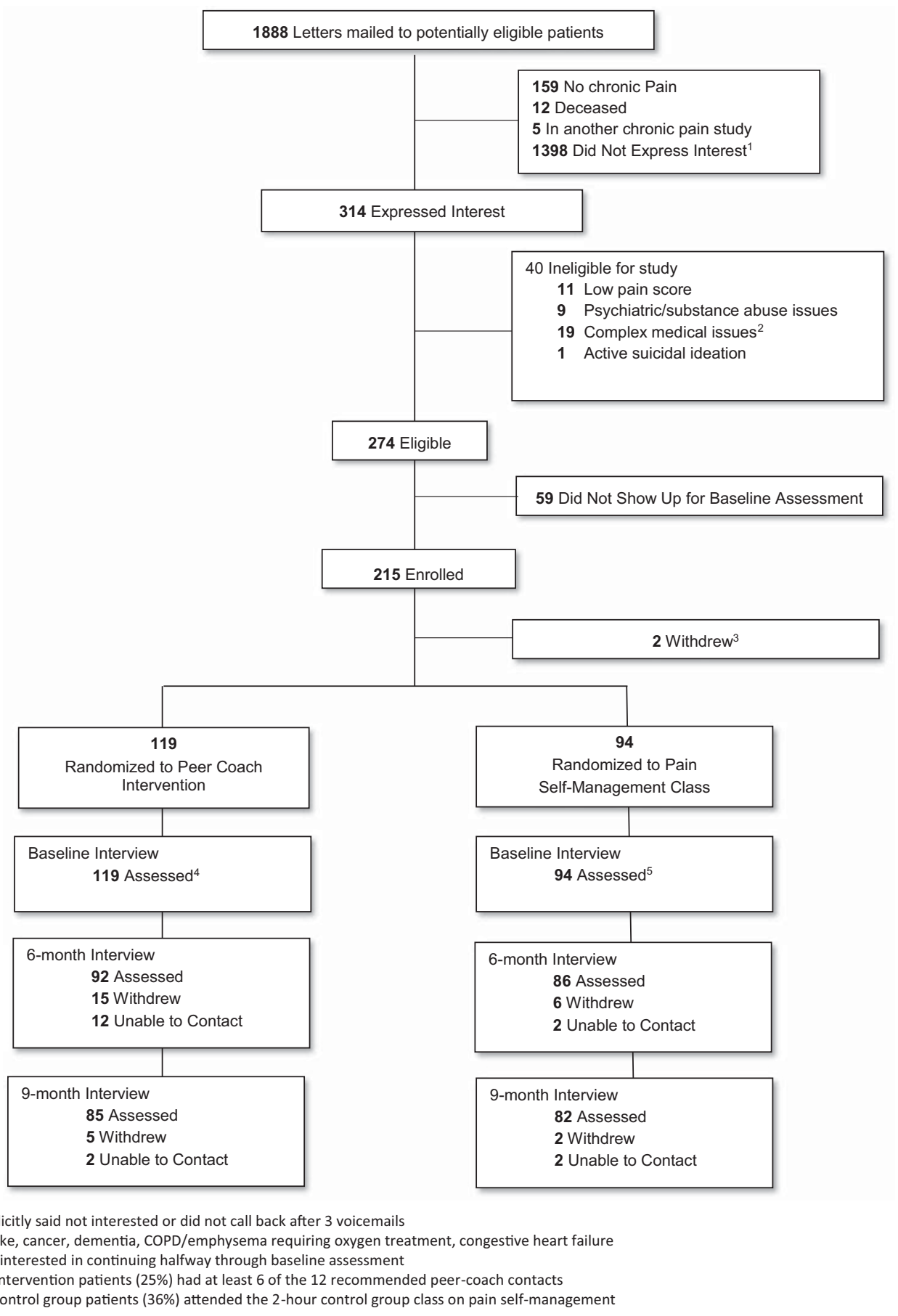

Fig. 1 Participant flow in ECLIPSE

hospitalizations, number of hospitalized days or opioids between intervention, and control patients during the 9-month study period (Appendix Table 1).

\section{Exploratory Findings}

Intervention dose. Patients $(N=30)$ who had at least 6 contacts with their peer coaches did not have significantly different outcomes at 6 or 9 months compared with the control group. The only significant changes in the intervention group were patient activation and pain catastrophizing. Patient activation increased from $54.4( \pm 11.3)$ at baseline to $60.8( \pm 13.2)$ at 6 months $(p=0.013)$ and to $59.7( \pm 15.4)$ at 9 months $(p$ $=0.039)$. Pain catastrophizing increased significantly from $17.0( \pm 9.6)$ to $21.6( \pm 13.0)$ from baseline to 6 months $(p=0.032)$ and then returned to near baseline at 9 months (18.5 \pm 14.3$)$. However, after adjusting for multiple comparisons, these changes were no longer significant. 


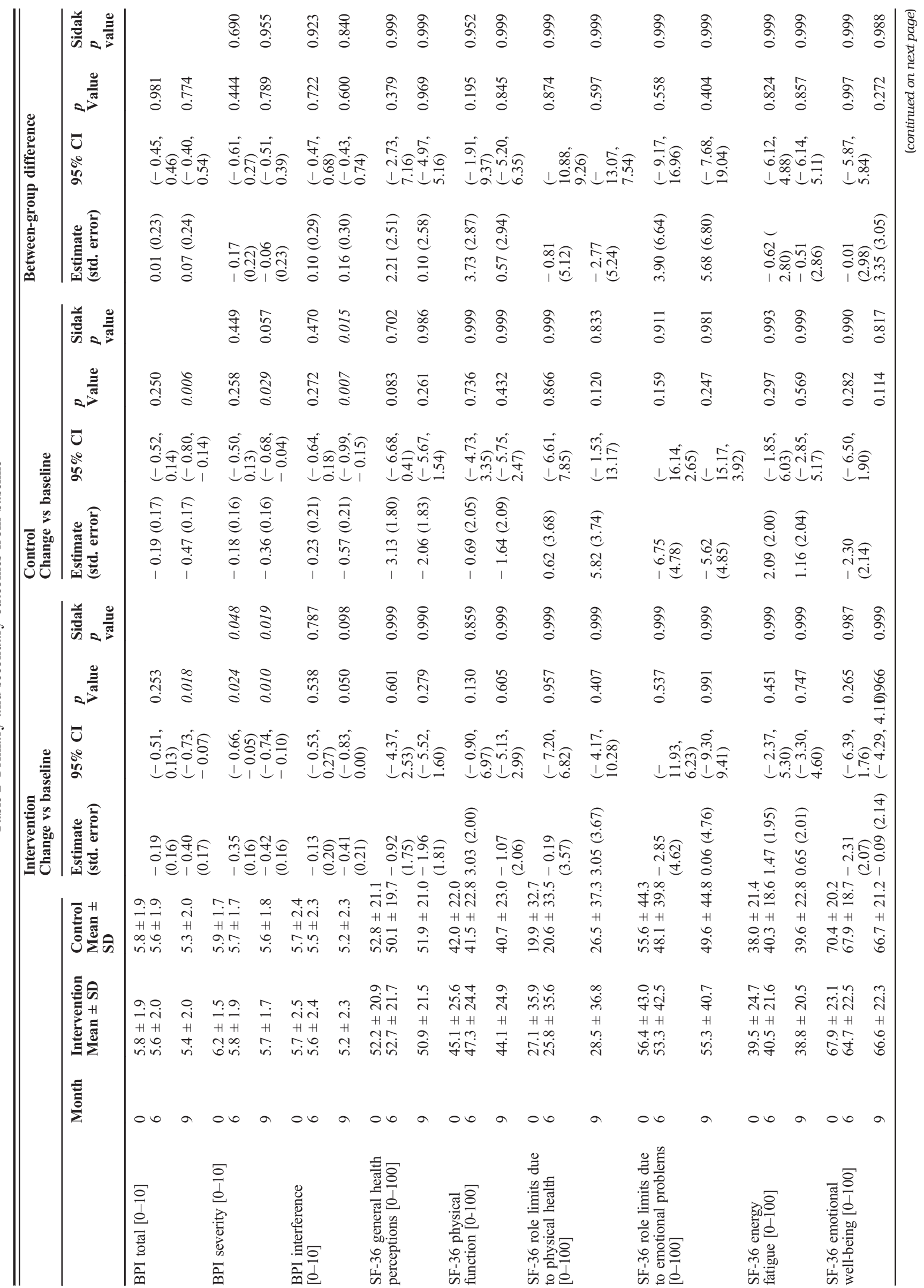




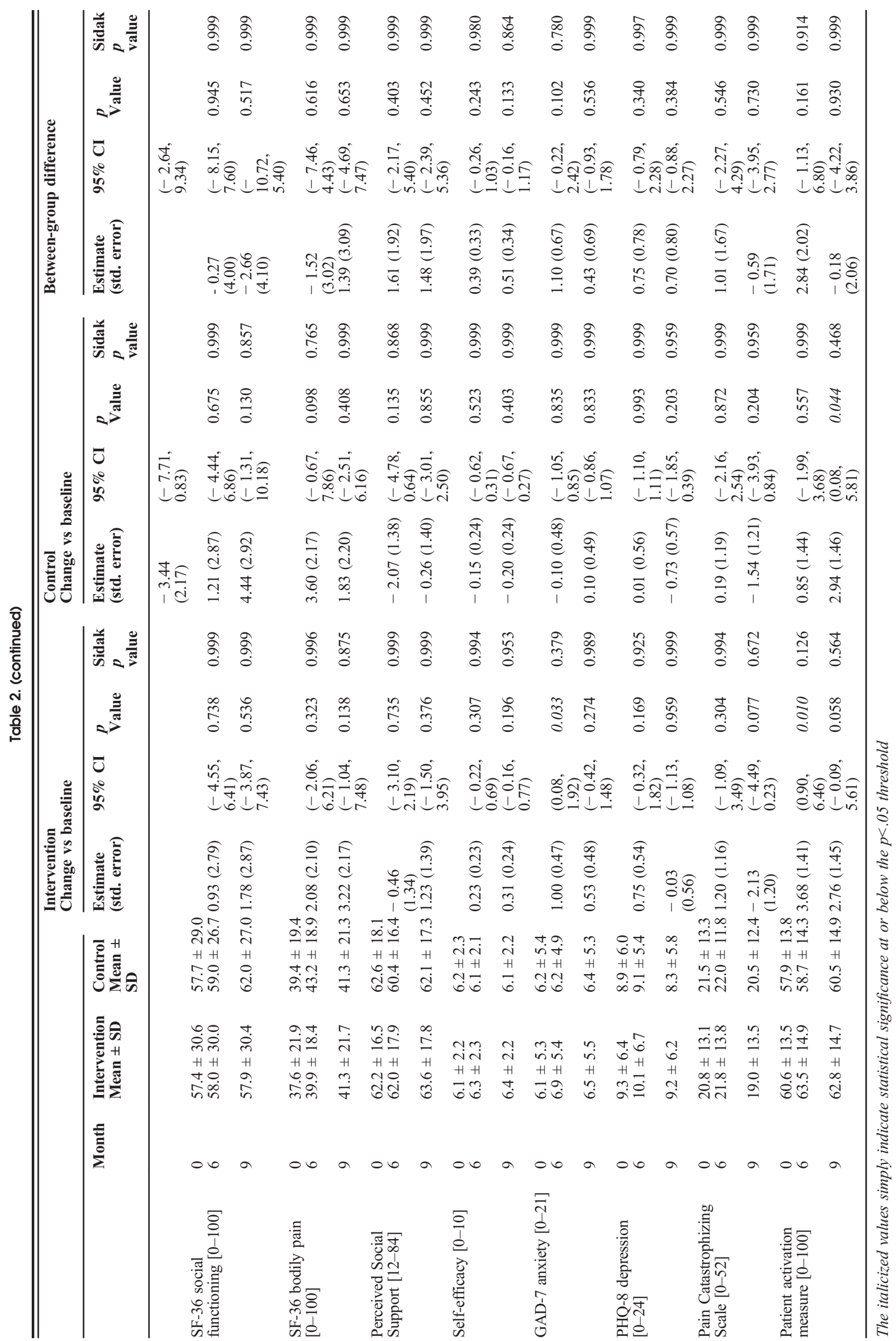


Moderation. Baseline patient activation was not found to be a significant moderator for any outcomes.

\section{DISCUSSION}

Our findings did not support the study hypotheses of improved pain among patients with chronic pain who participated in a 6month peer coaching intervention for chronic pain selfmanagement compared with a control group that was offered a 2-hour pain self-management class. Although pain (total BPI) among intervention participants decreased significantly from baseline to 9 months, this was seen in the control group as well. Even among the subgroup of participants who had at least 6 contacts with their coach (i.e., at least half of the recommend 12 contacts over 6 months), there were no significant differences between intervention and control.

Both the intervention and control groups experienced improvements in pain, which suggests that the 2-hour pain selfmanagement class offered to the control group might have had an effect. However, because only 34 control group participants (36\%) attended the pain self-management class, it is unlikely that these improvements are attributable to the class. It is unclear why both groups improved, although these improvements might be explained in part by natural fluctuations in symptoms, or simply regression to the mean. In addition, these small improvements which were well below the $30 \%$ reduction in pain considered to be clinically significant. ${ }^{18}$

In understanding these results, it is important to note that adherence to the intervention was quite low. Of the 84 patients for whom contact information is available, only $11(13 \%)$ reported the recommended 12 or more contacts with their coaches. Conversely, 54 (63\%) patients reported 5 or fewer coach contacts. Peer coaches were supervised throughout the trial, had regular booster sessions with the peer coach coordinator, and received regular one-on-one check-in calls, both to address any problems and to ensure that they were calling their assigned patients. Moreover, patients received regular calls to ensure that their coaches were contacting them. In cases where coaches were not making the required contacts, study staff followed up with the coach, and again with the patient. Similarly, when peer coaches could not reach their assigned patients, study staff made attempts to call the patients and encourage contact. Despite these efforts, engagement remained low. ECLIPSE was intentionally based on a volunteer model, in which peer coaches did not receive payment for coaching sessions, in an effort to test a model that would be easily implemented, with relatively low cost, in clinical settings. However, this trial suggests that relying on a volunteer model of peer support for patients with chronic pain might not result in patients receiving an adequate dose of the intervention.
Other studies suggest that compensation for peer coaches might be a more effective approach. For example, some successful peer coach interventions in diabetes provided generous stipends for the peer coaches, in some cases with the amount of monthly stipend tied to number of contacts they had with assigned participants. ${ }^{19-21}$ While exceptions can be found, such as a small study of peer support in heart failure, ${ }^{22}$ the majority of positive trials in the literature have used a model in which peers receive some kind of compensation.

However, because we did not systematically track peer coach outreach, we cannot determine whether the low number of reported contacts is attributable largely to failure of the peer coaches to initiate calls, or to difficulty reaching their patients when they tried to call. In any case, the low adherence speaks to an inherent challenge with behavioral interventions, which require more time and effort than other treatments, such as taking medication. Indeed, the National Academy of Medicine, while recognizing the importance of pain self-management, also recognized the inherent challenges associated with adherence. ${ }^{1}$ This is an important issue to explore in future research, given that poor adherence will negatively impact the effectiveness of behavioral interventions.

Given that results were not significant even for the 30 patients who had at least 6 contacts, low engagement might not fully explain our null findings, and, as a result, compensation of coaches might be less of an issue. It might be that peer support alone for pain self-management does not improve outcomes beyond standard care. Conversely, it is also possible that 6 meetings over 6 months were not a sufficient dose of the intervention to make a difference - prior successful peer support interventions averaged at least one contact every 2 weeks. Because engagement was low, we were not able to ascertain potential effects of the intervention for a higher number of contacts, and the optimal dose of such an intervention is unknown. As a result, we are unable to determine whether results are due to low engagement or simply to ineffectiveness of peer support in chronic pain.

This study is limited in that it was conducted at one site with veterans who were mostly male. Thus, results might not generalize to other patient groups or settings. Furthermore, healthcare utilization data was obtained from the VA's electronic medical record. We are unable to determine whether patients received healthcare from non-VA sources during the study. Finally, given the low dose of the intervention that the majority of participants received, it is difficult to draw conclusions on the effectiveness of the intervention if delivered at the prescribed dose. Future research should examine peer supported pain selfmanagement using salaried peer coaches, rather than volunteers. Such an approach would help to answer the question regarding the importance of peer coach compensation for adherence and effectiveness. The VA has such a model in mental health and is expanding the use of these peer specialists into primary care, creating the ideal environment for testing this model. 
Corresponding Author: Marianne S. Matthias, PhD; VA HSR\&D Center for Health Information and Communication, Roudebush VA Medical Center Indianapolis, IN, USA (e-mail: mmatthia@iupui.edu).

Funding Information This work was supported by the Department of Veterans Affairs Health Services Research and Development Merit Review Award to Dr. Matthias (IIR 14-070). The sponsor had no role in study design; in the collection, analysis, and interpretation of data; in the writing of the report; or in the decision to submit the article for publication. The views expressed in this article are those of the authors and do not necessarily represent the views of the Department of Veterans Affairs.

\section{Compliance with Ethical Standards:}

Conflicts of Interest: The authors declare that they do not have a conflict of interest.

\section{REFERENCES}

1. Institute of Medicine. Relieving pain in America: a blueprint for transforming prevention, care, education, and research. Washington, DC: National Academies Press; 2011.

2. Barlow J, Wright C, Sheasby J, Turner A, Hainsworth J. Selfmanagement approaches for people with chronic conditions: a review. Patient Educ Couns. 2010;48(2):177-187.

3. Bair MJ, Matthias MS, Nyland KA, et al. Barriers and facilitators to chronic pain self-management: a qualitative study among primary care patients with comorbid musculoskeletal pain and depression. Pain Med. 2009;10:1280-1290.

4. Matthias MS, Bair MJ, Nyland KA, et al. Self-management support and communication from nurse care managers compared to primary care physicians: a focus group study of patients with chronic musculoskeletal pain. Pain Manag Nurs. 2010;11(1):26-34.

5. Matthias MS, Miech EJ, Myers LJ, Sargent C, Bair MJ. An expanded view of self-management: patients' experiences with self-management education and support in an intervention for chronic musculoskeletal pain. Pain Med. 2012;13:1018-1028.

6. Dennis CL. Peer support within a health care context: a concept analysis. Int J Nurs Stud. 2003;40:321-332.

7. Heisler M, Vijan S, Makki F, Piette JD. Diabetes control with reciprocal peer support versus nurse care management: a randomized trial. Ann Intern Med. 2010;153(8):507-516.
8. Haltiwanger EP, Brutus $\mathbf{H}$. A culturally sensitive diabetes peer support for older Mexican-Americans. Occup Ther Int. 2011;19(2):67-75.

9. Piette J, Resnicow $\mathbf{K}$, Choi $\mathbf{H}$, Heisler $\mathbf{M}$. A diabetes peer support intervention that improved glycemic control: mediators and moderators of intervention effectiveness. Chronic Illn. 2013;9(4): 258-67.

10. Chinman M, Oberman RS, Hanusa BH, et al. A cluster randomized trial of adding peer specialists to intensive case management teams in the Veterans Health Administration. J Behav Health Serv Res. 2013;PMID 23657754 .

11. Salyers MP, Matthias MS, Spann CL, Lydick JM, Rollins AL, Frankel RM. The role of patient activation in psychiatric visits. Psychiatr Serv. 2009;60(11): 1535-1539.

12. Maeng DD, Martsolf GR, Scanlon DP, Christianson JB. Care coordination for the chronically ill: understanding the patient's perspective. Health Serv Res. 2012;47(5): 1960-1979.

13. Hibbard $\mathbf{J H}$, Greene $\mathbf{J}$. What the evidence shows about patient activation: better health outcomes and care experiences; fewer data on costs. Health Aff. 2013;32(2):207-214.

14. Matthias MS, Daggy J, Adams J, et al. Evaluation of a peer coach-led intervention to improve pain symptoms (ECLIPSE): rationale, study design, methods, and sample characteristics. Contemp Clin Trials. 2019;81:71-79.

15. Moerbeek M, Weng WK. Sample size formulae for trials comparing group and individual treatments in a multilevel model. Stat Med. 2008;27:28502864.

16. Bauer DJ, Sterba SK, Hallfors DD. Evaluating group-based interventions when control participants are ungrouped. Multivar Behav Res. 2008;43:210-236.

17. Matthias MS, Daggy J, Ofner S, McGuire AB, Kukla M, Bair MJ. Exploring peer coaches' outcomes: Findings from a clinical trial of patients with chronic pain. Patient Educ Couns. in press.

18. Miaskowski C, Dodd M, West C, et al. The use of a responder analysis to identify differences in patient outcomes following a self-care intervention to improve cancer pain management. Pain. 2007;129(1-2):55-63.

19. Heisler M, Choi H, Mase R, Long JA, Reeves PJ. Effectiveness of technologically enhanced peer support in improving glycemic control among predominantly African American, low-income adults with diabetes. Diabetes Educ. 2019;45(3):260-271.

20. Tang TS, Funnell M, Sinco B, Spencer MS, Heisler M. Peer-led, empowerment-based, approach to self-management efforts in diabetes (PLEASED): a randomized controlled trial in an African-American community. Ann Fam Med. 2015;13(Supplement 1):S27-35.

21. Long JA, Jahnle EC, Richardson DM, Loewenstein G, Volpp KG. Peer mentoring and financial incentives to improve glucose control in African American veterans. Ann Intern Med. 2012;156(6):416-424.

22. Riegel B, Carlson B. Is individual peer support a promising intervention for persons with heart failure? J Cardiovasc Nurs. 2004; 19(3):174- 183.

Publisher's Note: Springer Nature remains neutral with regard to jurisdictional claims in published maps and institutional affiliations. 